\title{
Importance of fingering convection for accreting white dwarfs in the framework of full evolutionary calculations: the case of the hydrogen-rich white dwarfs GD 133 and G 29-38
}

\author{
F. C. Wachlin ${ }^{1}$, G. Vauclair ${ }^{2,3}$, S. Vauclair ${ }^{2,3}$, and L. G. Althaus ${ }^{1}$ \\ ${ }^{1}$ Instituto de Astrofísica de La Plata (UNLP - CONICET), Facultad de Ciencias Astronómicas y Geofísicas, \\ Universidad Nacional de La Plata, B1900FWA La Plata, Argentina \\ e-mail: fcw@fcaglp.unlp.edu.ar \\ 2 Université de Toulouse, UPS-OMP, IRAP, 31400 Toulouse, France \\ 3 CNRS, IRAP, 14 avenue Édouard Belin, 31400 Toulouse, France
}

Received 18 November 2016/ Accepted 28 December 2016

\begin{abstract}
Context. A large fraction of white dwarfs show photospheric chemical composition that is polluted by heavy elements accreted from a debris disk. Such debris disks result from the tidal disruption of rocky planetesimals that have survived to whole stellar evolution from the main sequence to the final white dwarf stage. Determining the accretion rate of this material is an important step toward estimating the mass of the planetesimals and understanding the ultimate fate of the planetary systems.

Aims. The accretion of heavy material with a mean molecular weight, $\mu$, higher than the mean molecular weight of the white dwarf outer layers, induces a double-diffusive instability producing the fingering convection and an extra-mixing. As a result, the accreted material is diluted deep into the star. We explore the effect of this extra-mixing on the abundance evolution of $\mathrm{Mg}, \mathrm{O}, \mathrm{Ca}, \mathrm{Fe}$ and $\mathrm{Si}$ in the cases of the two well-studied polluted DAZ white dwarfs: GD 133 and G 29-38.

Methods. We performed numerical simulations of the accretion of material that has a chemical composition similar to the bulk Earth composition. We assumed a continuous and uniform accretion and considered a range of accretion rates from $10^{4} \mathrm{~g} / \mathrm{s}$ to $10^{10} \mathrm{~g} / \mathrm{s}$. Two cases are simulated, one using the standard mixing length theory (MLT) and one including the double-diffusive instability (fingering convection).

Results. The double-diffusive instability develops on a very short timescale. The surface abundance rapidly reaches a stationary value while the depth of the zone mixed by the fingering convection increases. In the case of GD 133, the accretion rate needed to reproduce the observed abundances exceeds by more than two orders of magnitude the rate estimated by neglecting the fingering convection. In the case of G 29-38 the needed accretion rate is increased by approximately 1.7 dex.

Conclusions. Our numerical simulations of the accretion of heavy elements on the hydrogen-rich white dwarf GD 133 and G 29-38 show that fingering convection is an efficient mechanism to mix the accreted material deeply. We find that when fingering convection is taken into account, accretion rates higher by 1.7 to 2 dex than those inferred from the standard MLT are needed to reproduce the abundances observed in G 29-38 and GD 133
\end{abstract}

Key words. white dwarfs - accretion, accretion disks - stars: abundances - stars: evolution - instabilities - stars: interiors

\section{Introduction}

Since the early discovery of an infra-red excess from the hydrogen-rich white dwarf G29-38 (Zuckerman \& Becklin 1987), the prototype of the DAZ class, it has become evident that a large fraction of white dwarfs are surrounded by debris disk from which heavy elements accrete at the white dwarf surfaces and pollute their chemical composition. These debris disks result from the disruption by tidal effects of planetesimal orbiting the white dwarfs (Jura 2003).

White dwarfs (WDs) surrounded by such debris disks, make up $56 \%$ of the sample of warm DA white dwarfs sample that has effective temperature $17000 \mathrm{~K} \leq T_{\text {eff }} \leq 27000 \mathrm{~K}$ analyzed by Koester et al. (2014). Zuckerman et al. (2010) estimate this fraction to be $\approx 1 / 3$ for the DB white dwarfs in the effective temperature range $13500 \mathrm{~K} \leq T_{\text {eff }} \leq 19500 \mathrm{~K}$.

To become disintegrated, the planetesimals must come close to, or cross through, the tidal radius of the white dwarf. To achieve this condition their original, presumably circular, orbits must be perturbed by gravitational interactions with one or more massive planets, of either Jovian or Neptunian sizes. This would cause them to evolve toward elliptical orbits so as to pass close enough to the white dwarf tidal radius. A number of scenarios of planetary systems evolution through the giant and asymptotic giant branches have demonstrated that massive planets may survive these phases of the star evolution and be able to perturb the planetesimal orbits to large eccentricity (e.g., Debes \& Sigurdsson 2002; Debes et al. 2012; Mustill et al. 2013; Veras et al. 2013, 2014b,a, 2015b,c,a, 2016; Veras 2016; Frewen \& Hansen 2014)

The recent discovery of planetesimal fragments transiting in front of the white dwarf WD $1145+017$ is evidence that disintegrations of planetesimals may indeed occur in such circumstances (Vanderburg et al. 2015; Croll et al. 2017; Gänsicke et al. 2016; Xu et al. 2016). The analysis of the chemical composition of the polluted white-dwarf atmospheres shows that the disrupted planetesimals are rocky bodies similar to the asteroids in the solar system. Their composition is mostly similar 
to the Earth bulk composition (Melis et al. 2011; Xu et al. 2014; Jura \& Young 2014).

The determination of the chemical composition of polluted white dwarfs offers a unique opportunity to scrutinize the composition of the disrupted planetesimals orbiting extrasolar planetary systems. However, in order to relate the chemical composition of the disrupted bodies to the composition observed at the white dwarf surface it is necessary to correctly describe the various processes which affect the material once accreted at the white dwarf surface. If there is a convective zone in the stellar outer layers, the accreted elements are first mixed throughout this zone. If not, they first accumulate in the photosphere. In both cases, they are further mixed in the extra-mixing zone that is triggered by the fingering convection, a process that we discuss in the present paper. In addition they settle downwards by gravitational settling through the bottom of the mixed zone. Previous estimates were based on the assumption that the accreted material diffuses downwards by gravitational settling alone (e.g., Farihi et al. 2012; Koester et al. 2014).

However, the double-diffusive instability resulting from the inverse $\mu$-gradient induced by the accretion of heavy elements is much more efficient that the gravitational settling and has to be taken into account. This kind of instability is well known in oceanography where it is referred to as the thermohaline convection. In the oceanographic context the instability occurs in situations where warm saltwater lies on top of cooler freshwater. It leads to the so-called salt fingers. The same instability has been found to occur in various astrophysical situations where an inverse $\mu$ gradient develops in a thermally stable layer (Vauclair 2004; Stancliffe et al. 2007; Stancliffe \& Glebbeek 2008; Thompson et al. 2008; Théado et al. 2009; Garaud 2011; Traxler et al. 2011; Wachlin et al. 2011; Théado \& Vauclair 2012; Vauclair \& Théado 2012; Brown et al. 2013; Wachlin et al. 2014; Zemskova et al. 2014; Deal et al. 2015). In the astrophysical context the instability is refereed to as fingering convection, and it is well known to lead to efficient extra-mixing.

Preliminary exploration of the effect of the fingering convection in the case of static white dwarf models accreting debris disk material has shown that ignoring this extra-mixing leads to underestimating the accretion rates by several orders of magnitude for DA (hydrogen-atmosphere) white dwarfs. By contrast it has negligible or marginal effect on the accretion rate on DB (helium-atmosphere) white dwarfs (Deal et al. 2013).

In the present paper, we extend the previous study by simulating the time dependent accretion of debris-disk material on two DA white dwarfs. We selected the two DAZ G 29-38, the prototype of this class, and GD 133. Both have an IR excess, signature of a debris disk (Zuckerman \& Becklin 1987; Reach et al. 2005; Jura et al. 2007, 2009; Farihi et al. 2009), have a heavily polluted atmosphere and, in addition, both are ZZ Ceti pulsators. Their parameters and abundances are rather well determined from spectroscopy (Xu et al. 2014) and asteroseismology (Chen \& Li 2013; Fu et al. 2016).

The paper is organized as follows. Section 2 indicates how we obtain the initial models for the simulations. In Sect. 3 we describe the numerical simulations and their results are given in Sect. 4. We summarize and discuss our results in Sect. 5.

\section{Initial models}

To assess the impact of fingering convection on the estimated accretion rates, we concentrate on two different models of hydrogen-rich (DA) white dwarfs. Although fingering convection has been shown to be a relevant physical process in accreting
Table 1. Stellar parameters adopted for G 29-38 and GD 133.

\begin{tabular}{ccccc}
\hline \hline Star & $M_{\mathrm{WD}}\left[M_{\odot}\right]$ & $T_{\mathrm{eff}}[\mathrm{K}]$ & $\log M_{\mathrm{H}} / M_{\mathrm{WD}}$ & $\log M_{\mathrm{cvz}} / M_{\mathrm{WD}}$ \\
\hline G 29-38 & 0.85 & $11820 \pm 100$ & $10^{-4}$ & -13.95 \\
GD 133 & 0.63 & $12400 \pm 100$ & $3.16 \times 10^{-5}$ & -16.19 \\
\hline
\end{tabular}

Notes. For G 29-38, the mass and the effective temperature are the values derived from spectroscopy (Xu et al. 2014). For GD 133, the values are derived from the asteroseismology analysis of Fu et al. (2016). They are in good agreement with the values given by spectroscopy (Xu et al. 2014). The hydrogen mass fractions are from asteroseismology: Chen \& Li (2013) for G 29-38, and Fu et al. (2016) for GD 133.

white dwarfs (Deal et al. 2013), the role of this process on the expected accretion rates in evolving white dwarf still remains to be investigated. In this paper we endeavour to investigate this, by analysing two white dwarfs: GD 133 and G 29-38. We based our study on a consistent treatment of mixing processes and whitedwarf evolution. We performed two different types of numerical experiment: one assuming pure gravitational settling and another that also includes fingering convection. Table 1 shows the adopted stellar parameters for both stars ${ }^{1}$.

Our initial white-dwarf models were taken from Renedo et al. (2010). These models are the result of full evolutionary calculations from the zero age main sequence, through the core hydrogen burning phase, the helium burning phase and the thermally pulsing asymptotic giant branch phase to the white dwarf stage. Specifically, we selected two models with stellar masses closest to that of our objects of interest: $M_{\mathrm{WD}}=0.632 M_{\odot}\left(Z=0.01, M=2.25 M_{\odot}\right.$ progenitor $)$, for GD 133, and $M_{\mathrm{WD}}=0.837 M_{\odot}\left(Z=0.01, M=4.0 M_{\odot}\right.$ progenitor), for G 29-38. These models were evolved from the beginning of the cooling branch to lower luminosities, allowing element diffusion to operate. As soon as heavier elements sunk, we artificially modified the envelope of the models in order to match the amount of hydrogen according to the asteroseismological inferences (Chen \& Li 2013; Fu et al. 2016). We evolved the models until they reached the final effective temperature listed in Table 1, where we stopped the simulations. The resulting models are considered as our initial models. From this stage, accretion was allowed to operate.

\section{Numerical computations}

All computations were done using the LPCODE stellar evolutionary code (Althaus et al. 2005, 2013, 2015), properly modified to simulate the accretion scenario and to follow the evolution of some chemical elements that were not present in the original version of the code. These chemical elements are ${ }^{24} \mathrm{Mg},{ }^{28} \mathrm{Si},{ }^{40} \mathrm{Ca}$, and ${ }^{56} \mathrm{Fe}$, with initial abundances in solar proportions. Gravitational settling was included following Burger's scheme (Burgers 1969). For details about our stellar code, and the input physics, we refer the reader to the above cited papers.

There has been some discussion about whether the accretion from the debris disk occurs as a continuous or a discontinuous process and whether the accreted material follows a particular geometry. In the case of G 29-38, Thompson et al. (2010) find a

1 For the particular case of G 29-38 there is a large variety of parameters estimated by different authors (see Romero et al. 2012; Chen \& Li 2013; $\mathrm{Xu}$ et al. 2014). We decided to use the asteroseismological parameters in Chen \& $\mathrm{Li}$ (2013) to study a more massive model and also to avoid the case of an unusually thin hydrogen envelope such as that estimated by Romero et al. (2012). 
suggestion that the accretion geometry could be time dependent, pointing to a discontinuous accretion producing spots of heavy elements on the white-dwarf surface (see also Montgomery et al. 2008) and to the influence of a potential magnetic field on the accretion process. However, the time variability of the heavy element abundances, $\mathrm{Ca}$ in the case of G 29-38, which would be the signature of such a discontinuous accretion, was not confirmed (Debes \& López-Morales 2008). In addition, GD 133 and G 2938 have no detected magnetic field (Ferrario et al. 2015).

In the present simulations, accretion was implemented as a continuous process and the accreted material is assumed to be uniformly distributed on the star's surface. We performed simulations for different accretion rates, ranging from $10^{4} \mathrm{~g} / \mathrm{s}$ to $10^{10} \mathrm{~g} / \mathrm{s}$. Metal abundances of accreted matter were set to mimic the composition of the bulk Earth (Allègre et al. 2001). The amount of accreted matter was mixed instantaneously in the convective zone, by assuming that the mixing timescale is much shorter than the characteristic cooling timescale.

Our computations were done in the framework of the double diffusion theory (GNA theory) developed by Grossman et al. (1993), adopting the prescription of Brown et al. (2013) for the diffusion coefficient of fingering convection zones. This formulation allows the treatment of the fingering convection instability (for more details, see Wachlin et al. 2011). We also performed computations using the classical convection theory (ML2, with $\alpha=0.8$ ). Whereas fingering convection is included in the GNA computations, it is not taken into account in the simple mixing length theory (MLT) method. For numerical purposes, small evolutionary time steps were adopted, particularly for large accretion rates. Specifically, we used time steps of the orders of days for ML2 simulations and of the order of hours, for GNA experiments.

\section{Results}

Time evolution of surface composition for the model representative of GD 133, once accretion has been turned on, is displayed in Fig. 1 for the various adopted values of the accretion rate. For the sake of clarity we only show one element, namely, ${ }^{56} \mathrm{Fe}$. The situation for other heavy elements is similar, although each one reaches its own final abundance level according to its relative presence in the accreted matter and to its individual diffusion velocity. From Fig. 1 we see that, for the same accretion rate, the surface contamination becomes larger when fingering convection is disregarded. This is because the accreted material mixes almost instantaneously in the convective zone, thus leading to the formation of a chemical inversion (heavier material on top of lighter material) at its base. In the framework of the canonical convection theory (MLT), this chemical inversion does not alter the chemical structure in deeper layers; there, abundance changes are only due to gravitational settling. In contrast, when the GNA convection theory is considered, the chemical inversion triggers the appearance of fingering convection below the convective zone. As a consequence, heavy elements become more efficiently mixed into deeper layers, reducing their abundances in the atmosphere (mainly by dilution). Diffusion may also contribute to lower the metal contamination of the atmosphere but, as compared with the efficiency of fingering convection, its contribution is much smaller. Figure 1 clearly illustrates that a stationary state is rapidly reached for all the adopted accretion rates. In fact, less than ten years of accretion is needed for the surface abundances to be stabilized. In the case of the MLT, shorter times are needed to reach a stationary state.

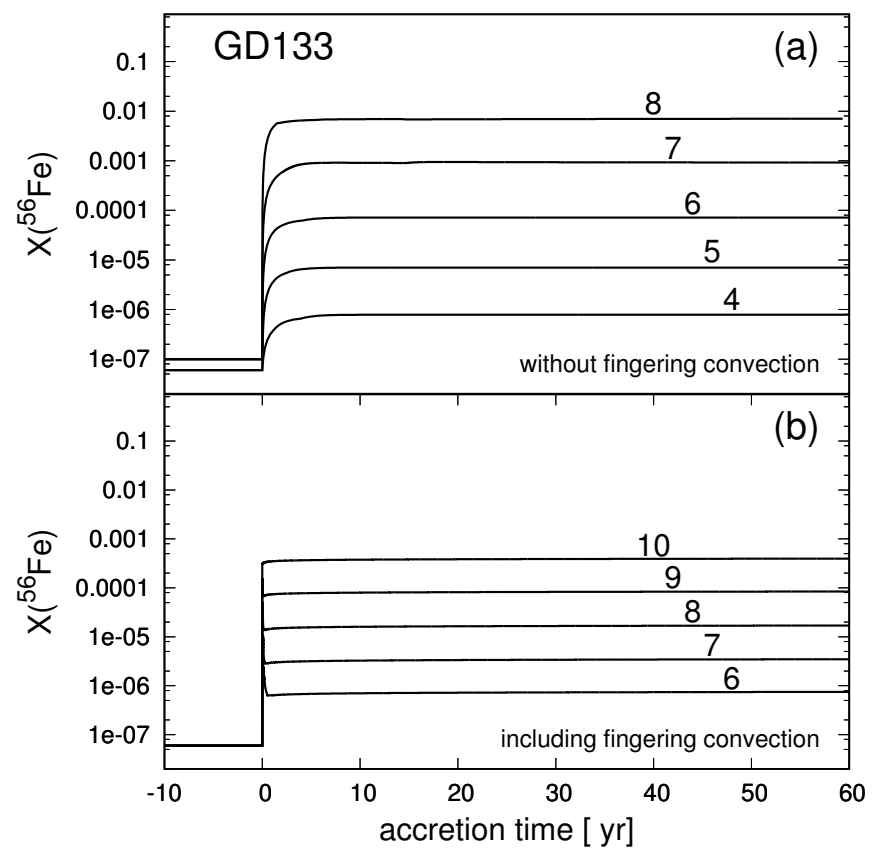

Fig. 1. Time evolution of ${ }^{56} \mathrm{Fe}$ surface abundance for the model of GD 133. Top panel: without taking fingering convection into account. Bottom panel: including fingering convection. Numbers indicate the logarithm of the adopted accretion rates, in units of $\mathrm{g} / \mathrm{s}$.

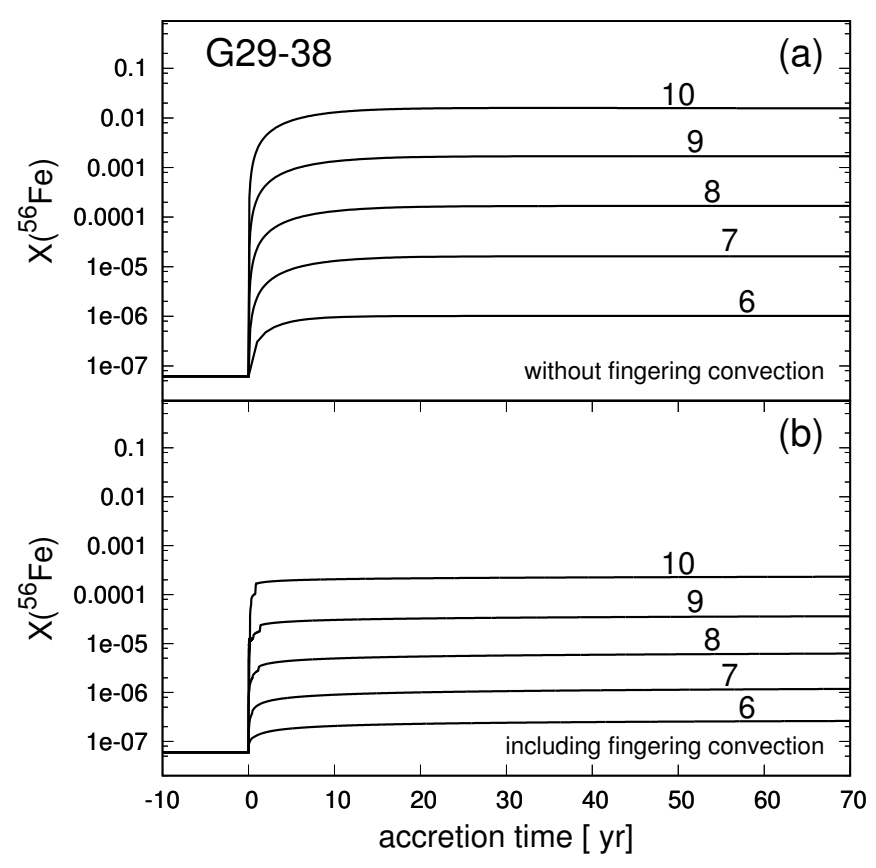

Fig. 2. Same as Fig. 1 for G 29-38.

The results for G 29-38 are depicted in Fig. 2. As compared with the situation in GD 133, longer times are needed to reach the stationary state. We also note that, when fingering convection is not considered, much larger accretion rates (almost two orders of magnitude) are needed in G 29-38 than in GD 133 to reach a given surface contamination. This is related to the depth reached by the convective region, which is about a hundred times more massive for G 29-38 than for GD 133, as can be seen in Table 1. 


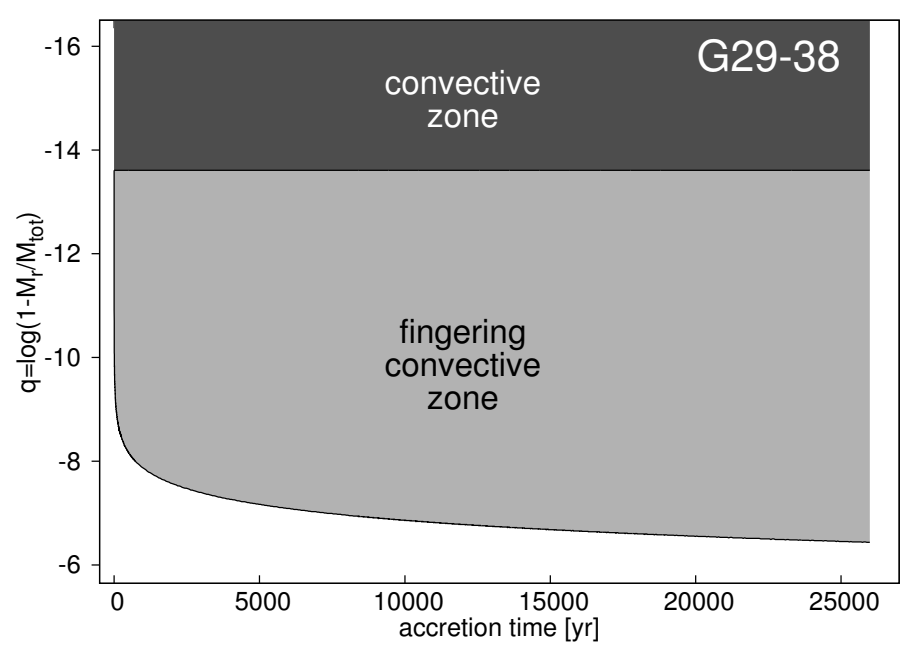

Fig. 3. Time evolution of the stellar structure for the G 29-38 model. The accretion rate was of $10^{10} \mathrm{~g} / \mathrm{s}$.

This is different when fingering convection is allowed, because it mixes matter deeper inside the star.

Figure 3 shows the behavior of fingering convection in the presence of accretion for our maximum accretion rate $\left(10^{10} \mathrm{~g} / \mathrm{s}\right)$ for $\mathrm{G} 29-38$. We note that the fingering convection zone grows very rapidly once accretion has started. Over a few centuries, it spans a region which embraces about 1000 times more mass than the convective zone. This strongly dilutes heavy elements, lowering surface contamination. It is somewhat surprising that the fingering convective zone was still evolving deeper after more than $25000 \mathrm{yr}$ of continuous accretion. It would have been interesting to follow this experiment until a steady state could be reached but unfortunately we had to stop the simulation because of the (prohibitively) long run time required (Fig. 3 demanded one month of cpu time). In any case, it is evident that the fingering convection zone goes on growing for a period of time substantially longer than the time needed for surface elements to reach a stationary state. A similar situation was also observed for the GD 133 case. The whole set of simulations shows that the higher the accretion rate the larger the fingering convection region. In this sense, Fig. 3 illustrates the situation of maximum depth reached by this zone in our simulations.

Figure 4 shows the internal chemical profiles of the last computed model in Fig. 3 for $\mathrm{H},{ }^{4} \mathrm{He},{ }^{16} \mathrm{O},{ }^{24} \mathrm{Mg},{ }^{28} \mathrm{Si},{ }^{40} \mathrm{Ca}$, and ${ }^{56} \mathrm{Fe}$. The dark gray region marks the dynamical convective envelope, in which the elements are almost instantaneously mixed and thus distributed homogeneously. Below this zone, there is a very extensive fingering convection region (light gray), where heavy elements are carried down to the interior layers by milder mixing, whose efficiency decreases toward the bottom of the fingering convective region. For this reason, this region shows chemical profiles where heavy-element abundances decrease with depth. We note that the fingering convective zone never reaches the compositional gradients inside the stellar core. Such a situation can, however, occur in the case of a very small hydrogen envelope. Since the maximum depth reached by the fingering convective zone is proportional to the accretion rate and considering that Fig. 4 shows the situation for the largest accretion rate, it is clear that none of our simulations was able to lead to element dredge-up from the deeper regions.

Figure 5 summarizes the final outcome of the simulations for our GD 133 model. Abundances are given as in Xu et al. (2014) for the purpose of comparison. The figure shows the final (steady

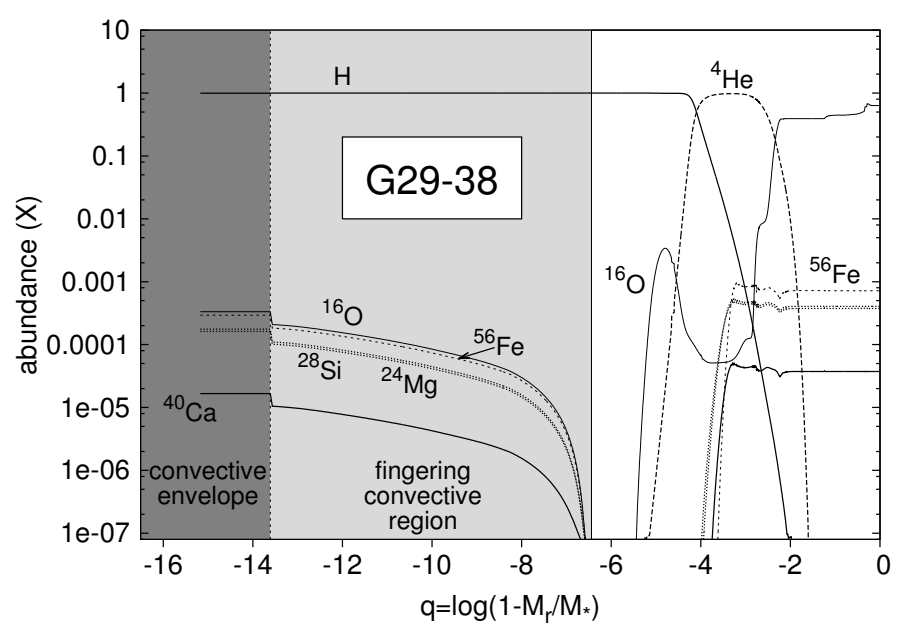

Fig. 4. Final chemical profile for our G 29-38 model submitted to an accretion rate of $10^{10} \mathrm{~g} / \mathrm{s}$. The lines corresponding to ${ }^{24} \mathrm{Mg}$ and ${ }^{28} \mathrm{Si}$ almost overlap. The same happens to ${ }^{16} \mathrm{O}$ and ${ }^{56} \mathrm{Fe}$. The bottom of the fingering convection zone never reaches central heavy elements.

state) surface abundances obtained by our simulations as a function of the accretion rate. From Fig. 5 we see that there is an almost linear relation between $[\mathrm{X} / \mathrm{H}]$ and the logarithm of accretion rates. The abundances grow faster when fingering convection is not taken into account, as shown by the different slopes of lines in the figure. Also included in Fig. 5 are some points (with error bars) corresponding to the observed abundances of a few elements reported by Xu et al. (2014) for GD 133, and overlap them with the lines of the corresponding element. If fingering convection is not considered, the observed points group themselves around $\log (\mathrm{d} M / \mathrm{d} t) \approx 5.5$ (for $[\mathrm{d} M / \mathrm{d} t]$ in $\mathrm{g} / \mathrm{s}$ ) while if fingering convection is taken into account, points are compatible with a much larger accretion rate, roughly around $\log (\mathrm{d} M / \mathrm{d} t) \approx 8$. Hence, neglecting fingering convection may lead to a difference of more than two orders of magnitude when estimating the accretion rate for the case of GD 133.

Figure 6 shows the situation for G 29-38, with some evident differences from GD 133. Perhaps the most conspicuous difference is that the dependence of the final abundance on the accretion rate is less steep here than in GD 133. This can be understood on the basis that G 29-38 has a convective zone that is 100 times larger (in mass) than GD 133. This introduces a large difference in the resulting contamination between the two stars when fingering convection is not considered. On the contrary, there is almost no such difference when fingering convection is considered (compare Figs. 5 and 6). The final surface abundances depend mainly on the total size of the envelope's mixed region and not on the original size of the convective zone.

Figure 6 also includes a few points with error bars, corresponding to the abundances reported by Xu et al. (2014) for G 29-38. We see that according to our "no fingering" simulations, the observed abundances should result from an accretion process at a rate of about $\log (\mathrm{d} M / \mathrm{d} t) \approx 7.8$. In contrast, numerical experiments including fingering convection suggest that the accretion rate should be $\operatorname{larger}, \operatorname{around} \log (\mathrm{d} M / \mathrm{d} t) \approx 9.5$. Thus, for G 29-38 we see that the difference between both estimates is smaller than for GD 133, namely, of approximately 1.7 dex. In any case, we find that the inclusion of fingering convection implies much larger accretion rates to explain observed abundances than inferences based on the standard MLT. 


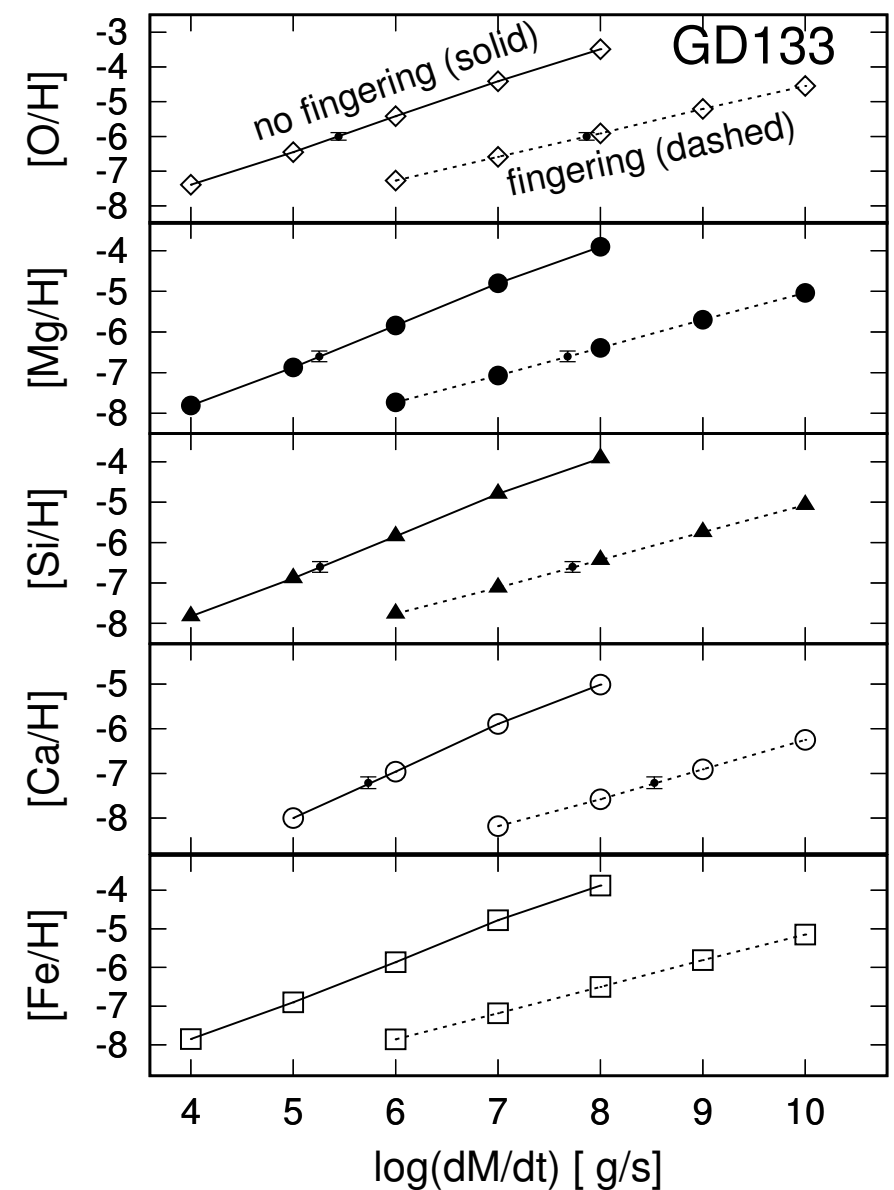

Fig. 5. Dependence of final abundances on the accretion rates and on the adopted convection theory for the case of GD 133. Solid lines stand for results obtained by using the classical mixing length convection theory (MLT). Dashed lines connect points obtained by implementing a double diffusion convection theory (GNA). Points with error bars refer to the observed abundances reported by Xu et al. (2014). For GD 133, $\mathrm{Fe}$ was not detected by Xu et al. (2014), thus no point was added in the corresponding figure. $[\mathrm{X} / \mathrm{H}]=\log n(\mathrm{X}) / n(\mathrm{H})$, the logarithmic number ratio of the abundance of element $\mathrm{X}$ relative to the abundance of $\mathrm{H}$.

\section{Summary and discussion}

We have performed numerical simulations of a continuous and uniform accretion of material with chemical composition similar to the bulk Earth composition onto the two DAZ and ZZ Ceti pulsators GD 133 and G 29-38. These two stars have been selected because they are both surrounded by a debris disk revealed by their infrared excess, have a detailed heavy element abundance analysis and have well constrained global parameters, including their hydrogen mass fraction, from asteroseismology.

Accretion rates ranging from $10^{4} \mathrm{~g} / \mathrm{s}$ to $10^{10} \mathrm{~g} / \mathrm{s}$ have been used in these simulations. Two cases have been considered: in the first case the accreted material was mixed in the surface convection zone, described by the standard mixing-length theory (MLT), and diffused through its bottom by gravitational settling, while in the second case we also introduced the double-diffusive instability induced by the building of the inverse $\mu$-gradient resulting from the accretion of heavy elements on top of the hydrogen-rich outer layers. In this second case, the instability is efficient in producing an extra-mixing by fingering convection. As a consequence, the accreted material is mixed in a much deeper region of the star. The resulting surface abundances of the

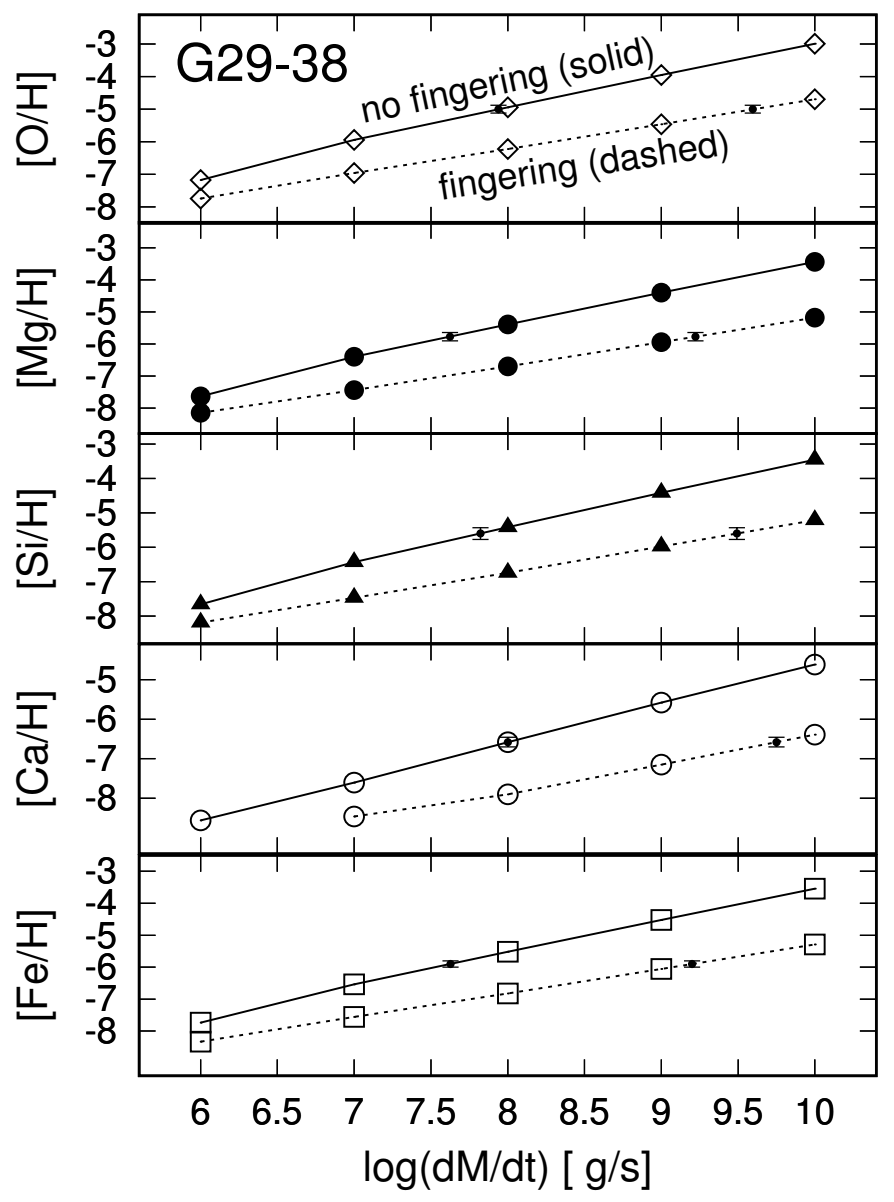

Fig. 6. Same as Fig. 5 but for G 29-38.

heavy elements which have been considered in this study $(\mathrm{Mg}$, $\mathrm{O}, \mathrm{Ca}, \mathrm{Fe}, \mathrm{Si}$ ) very rapidly reach a stationary value while the fingering convection regularly progress downwards. As a result of the extra-mixing the accretion rates needed to reproduce the abundances derived from the observations significantly exceed the rates estimated when the fingering-convection is ignored. In the case of GD 133, we find that the accretion rate required to reproduce the observed abundances is $\log (\mathrm{d} M / \mathrm{d} t) \approx 5.5$ (for $[\mathrm{d} M / \mathrm{d} t]$ in $\mathrm{g} / \mathrm{s})$ when fingering convection is not taken into account, but rises to $\log (\mathrm{d} M / \mathrm{d} t) \approx 8$ when fingering convection is properly taken into account. For G $29-38$ the observed abundances should result from an accretion rate $\log (\mathrm{d} M / \mathrm{d} t) \approx 7.8$ according to our no fingering simulations but an accretion rate around $\log (\mathrm{d} M / \mathrm{d} t) \approx 9.5$ is needed when fingering convection is included.

We conclude that the extra-mixing induced by the fingering convection is an important effect which must be taken into account when estimating the accretion rates needed to reproduce the heavy element abundances observed in the DAZ white dwarfs. It may imply that significantly more massive rocky bodies may have been disrupted by tidal effects than previously estimated.

Acknowledgements. Part of this work was supported by the Agencia Nacional de Promoción Científica y Tecnológica (ANPCyT) through the Programa de Modernización Tecnolǵica BID 1728/OC-AR, by the PIP 112-200801-00940 grant from CONICET (Argentina). Some simulations were run on IFLySiB's Cluster, which is part of SNCAD-MinCyT, Argentina. 


\section{References}

Allègre, C., Manhès, G., \& Lewin, É. 2001, Earth Planet. Sci. Lett., 185, 49

Althaus, L. G., Serenelli, A. M., Panei, J. A., et al. 2005, A\&A, 435, 631

Althaus, L. G., Miller Bertolami, M. M., \& Córsico, A. H. 2013, A\&A, 557, A19

Althaus, L. G., Camisassa, M. E., Miller Bertolami, M. M., Córsico, A. H., \& García-Berro, E. 2015, A\&A, 576, A9

Brown, J. M., Garaud, P., \& Stellmach, S. 2013, ApJ, 768, 34

Burgers, J. M. 1969, Flow Equations for Composite Gases (New York: Academic Press)

Chen, Y.-H., \& Li, Y. 2013, Res. Astron. Astrophys., 13, 1438

Croll, B., Dalba, P. A., Vanderburg, A., et al. 2017, ApJ, 836, 82

Deal, M., Deheuvels, S., Vauclair, G., Vauclair, S., \& Wachlin, F. C. 2013, A\&A, 557, L12

Deal, M., Richard, O., \& Vauclair, S. 2015, A\&A, 584, A105

Debes, J. H., \& López-Morales, M. 2008, ApJ, 677, L43

Debes, J. H., \& Sigurdsson, S. 2002, ApJ, 572, 556

Debes, J. H., Walsh, K. J., \& Stark, C. 2012, ApJ, 747, 148

Farihi, J., Jura, M., \& Zuckerman, B. 2009, ApJ, 694, 805

Farihi, J., Gänsicke, B. T., Wyatt, M. C., et al. 2012, MNRAS, 424, 464

Ferrario, L., de Martino, D., \& Gänsicke, B. T. 2015, Space Sci. Rev., 191, 111

Frewen, S. F. N., \& Hansen, B. M. S. 2014, MNRAS, 439, 2442

Fu, J.-N., Vauclair, G., Su, J., et al. 2016, in 20th European Workshop on White Dwarfs, ASP Conf. Ser., 509, 355

Gänsicke, B. T., Aungwerojwit, A., Marsh, T. R., et al. 2016, ApJ, 818, L7

Garaud, P. 2011, ApJ, 728, L30

Grossman, S. A., Narayan, R., \& Arnett, D. 1993, ApJ, 407, 284 Jura, M. 2003, ApJ, 584, L91

Jura, M., \& Young, E. D. 2014, Ann. Rev. Earth Planet. Sci., 42, 45

Jura, M., Farihi, J., \& Zuckerman, B. 2007, ApJ, 663, 1285

Jura, M., Farihi, J., \& Zuckerman, B. 2009, AJ, 137, 3191

Koester, D., Gänsicke, B. T., \& Farihi, J. 2014, A\&A, 566, A34

Melis, C., Farihi, J., Dufour, P., et al. 2011, ApJ, 732, 90
Montgomery, M. H., Thompson, S. E., \& von Hippel, T. 2008, ApJ, 685, L133 Mustill, A. J., Villaver, E., Veras, D., Bonsor, A., \& Wyatt, M. C. 2013, EPJ Web Conf., 47, 06008

Reach, W. T., Kuchner, M. J., von Hippel, T., et al. 2005, ApJ, 635, L161

Renedo, I., Althaus, L. G., Miller Bertolami, M. M., et al. 2010, ApJ, 717, 183

Romero, A. D., Córsico, A. H., Althaus, L. G., et al. 2012, MNRAS, 420, 1462 Stancliffe, R. J., \& Glebbeek, E. 2008, MNRAS, 389, 1828

Stancliffe, R. J., Glebbeek, E., Izzard, R. G., \& Pols, O. R. 2007, A\&A, 464, L57 Théado, S., \& Vauclair, S. 2012, ApJ, 744, 123

Théado, S., Vauclair, S., Alecian, G., \& LeBlanc, F. 2009, ApJ, 704, 1262

Thompson, I. B., Ivans, I. I., Bisterzo, S., et al. 2008, ApJ, 677, 556

Thompson, S. E., Montgomery, M. H., von Hippel, T., et al. 2010, ApJ, 714, 296

Traxler, A., Garaud, P., \& Stellmach, S. 2011, ApJ, 728, L29

Vanderburg, A., Johnson, J. A., Rappaport, S., et al. 2015, Nature, 526, 546

Vauclair, S. 2004, ApJ, 605, 874

Vauclair, S., \& Théado, S. 2012, ApJ, 753, 49

Veras, D. 2016, Royal Society Open Science, 3, 150571

Veras, D., Mustill, A. J., Bonsor, A., \& Wyatt, M. C. 2013, MNRAS, 431, 1686

Veras, D., Jacobson, S. A., \& Gänsicke, B. T. 2014a, MNRAS, 445, 2794

Veras, D., Leinhardt, Z. M., Bonsor, A., \& Gänsicke, B. T. 2014b, MNRAS, 445, 2244

Veras, D., Eggl, S., \& Gänsicke, B. T. 2015a, MNRAS, 452, 1945

Veras, D., Eggl, S., \& Gänsicke, B. T. 2015b, MNRAS, 451, 2814

Veras, D., Leinhardt, Z. M., Eggl, S., \& Gänsicke, B. T. 2015c, MNRAS, 451, 3453

Veras, D., Mustill, A. J., Gänsicke, B. T., et al. 2016, MNRAS, 458, 3942

Wachlin, F. C., Miller Bertolami, M. M., \& Althaus, L. G. 2011, A\&A, 533, A139

Wachlin, F. C., Vauclair, S., \& Althaus, L. G. 2014, A\&A, 570, A58

Xu, S., Jura, M., Koester, D., Klein, B., \& Zuckerman, B. 2014, ApJ, 783, 79

Xu, S., Jura, M., Dufour, P., \& Zuckerman, B. 2016, ApJ, 816, L22

Zemskova, V., Garaud, P., Deal, M., \& Vauclair, S. 2014, ApJ, 795, 118

Zuckerman, B., \& Becklin, E. E. 1987, Nature, 330, 138

Zuckerman, B., Melis, C., Klein, B., Koester, D., \& Jura, M. 2010, ApJ, 722, 725 